\title{
The catastrophic antiphospholipid antibody syndrome: an unusual presentation of spontaneous acute intracranial haemorrhage
}

\begin{abstract}
Background: Catastrophic Antiphospholipid syndrome (CAPS) is a scarcely understood entity with a high mortality approaching $50 \%$ The rapid deterioration is triggered by a deadly cytokine storm, which is difficult to recognize and almost impossible to treat in time. Various regimens have been advocated, which include administration of monoclonal anti-phospholipid antibodies and pulses of high dose methyl prednisolone. However, these treatment modalities are only effective if the disease is diagnosed early and instituted.

Clinical presentation: We present an unusual case of multiple spontaneous intracranial haematomas in a young individual, with evidence of severe refractory coagulopathy in a background of multisystem dysfunction. A retrospective analysis of the subsequent rapid deterioration and resultant fatality provided us with important learning points for early diagnosis and management of triggers which can initiate and lead to a cascade of events that eventually end in death.

Conclusion: CAPS is a fatal disease which should be considered when dealing with spontaneous intracerebral bleeds with multisystem dysfunction in the background of coagulation derangements. Rapid treatment with steroids and monoclonal antibodies may be helpful in halting the progression of the disease and eventually help in repressing a fatal outcome. Identifying the trigger factors, that usually initiate the downhill cascade, are a priority, and avoiding such provocative factors is an important aspect of management. This will help in the prevention of morbidity and eventual mortality in such patients.
\end{abstract}

Keywords: Catastrophic Antiphospholipid Syndrome, Spontaneous Acute Intracranial Haemorrhage, Refractory coagulopathy, Intracranial haematomas, Spontaneous intracerebral bleeds
Volume 3 Issue 4 - 2015

\author{
Sibhi Ganapathy, Rajesh Parameshwaran Nair, \\ Anmol Nagaraj, Girish Menon R \\ Department of Neurosurgery, Manipal University, India
}

\begin{abstract}
Correspondence: Dr. Rajesh Nair, Neurosurgery Registrar, Department of Neurosurgery, Kasturba Hospital, Manipal University, Manipal 576104, India, Email rajeshnair39@yahoo.com
\end{abstract}

Received: November 25, 2015 | Published: December 04, 2015

\section{Case report}

A 42 year-old gentleman has presented to us with a low backache radiating to bilateral lower limbs since the past 2 years. It had been gradually progressive and has not been improved even with analgesics and bed rest. A detailed medical history was sought and he was found to have multiple co-morbid illnesses, as listed below:

- He was diagnosed to be hypothyroid in 2004 and started on replacement therapy, however, he has been non-compliant for the last 2 years.

- He was also detected to have spontaneous bilateral lower limb deep venous thrombosis in 2004 for which he was treated with anticoagulants. He discontinued his medication once the acute symptoms had tided over. The DVT progressed into secondary varicose veins and he eventually developed venous ulcers.

- He also complained of recurrent oral ulceration with recurrent bouts of laryngitis and pharyngitis.

- When evaluated in 2014, he was detected to have an elevated creatinine level without oliguria. He was advised further evaluation of this derangement in his renal function tests, but deferred the same.

A neurological evaluation was unremarkable and radio-imaging of the lumbo-sacral spine was requested. He was found to have a L4-5 Grade 1 spondylolisthesis with L4 spondylolysis with a pseudodisc at L4-5. Conservative management was advocated and he improved with bed rest and muscle relaxants.
His blood work up, had shown a grossly elevated aPTT (activated plasma-thromboplastin test) and PT/INR (Prothrombin time, International normalized ratio). He was advised to have a complete evaluation of his coagulation profile since he had multisystem dysfunction despite discontinuing the anticoagulants prescribed to him. He refused the same and, the following day, was discharged on request.

His second admission to the hospital was a month later and he had presented to us with severe headache, vomiting and altered sensorium. He seemed to open eyes to call and obey simple commands, however, he seemed to be drowsy. Fundus examination showed papilloedema and CT Brain (Figure 1) showed a moderate size subacute subdural in the right fronto-temporo-parietal region with a small left occipital cystic extradural collection. There was diffuse cerebral oedema with partial effacement of the basal cisterns, an ominous sign of raised intracranial pressure (ICP).He was started on antiepileptics and analgesics with no significant improvement in his sensorium.

Before confirmatory workup could be assessed, he developed severe bradycardia (heart rate of 42 beats per minute). The danger of imminent neurological collapse forced us to intervene despite the high risk of rebleed. Under the cover of plasma products, multiple burr holes and evacuation of the right subacute subdural haematoma was done. Post procedure, the patient was stable. Glasgow coma scale was E3M6V4 with bilateral pupils being equal and sluggishly reacting to light.

The post op computed tomographic scan of the brain (CT Brain) was satisfactory with complete evacuation of the bleed, however 
the oedemapersisted (Figure 2a). Coagulation studies remained deranged inspite of corrective measures. An inhibitor screen showed the presence of circulating inhibitors, but all other immunological markers were negative.

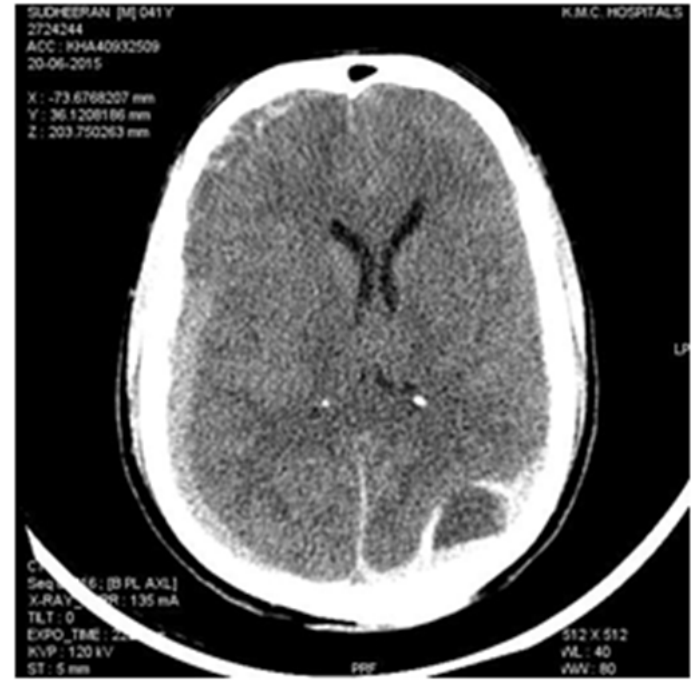

Figure I Preoperative CT Brain plain showing a right fronto-temporoparietal acute on chronic SDH with mass effect and midline shift with a left parieto-occipital extradural bleed.

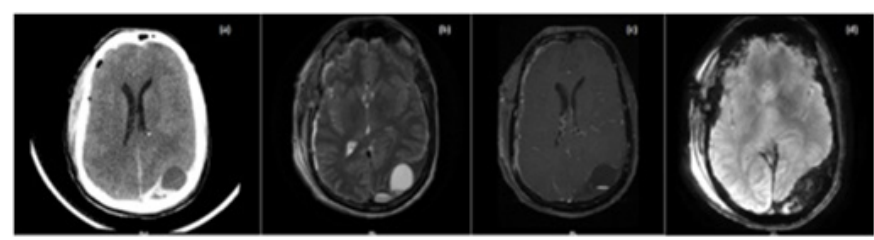

Figure 2 Postoperative scan, (a) Plain, CT Brain showing postop status with evacuation of the right acute on chronic SDH with a persistent left parietooccipital EDH bleed. MRI Brain, (b) T2W series showing a? cystic extradural collection in the left PO region with minimal residual right FTP SDH, with no contrast enhancement (c) and blooming $n \mathrm{SWI}$ (d) suggestive of an extradural haematoma.

A subsequent MRI of the brain showed no lesions, AVMs, or features of meningitis (Figure 12b,c,d). Coagulation studies were severely deranged with a new onset thrombocytopenia. Neurological Behcet'sdisease or an overlap-collagen vascular disorder, were among the clinical differentials being considered. Blood investigations were sent for APLA and a few other factors, including c-ANCA and ANA.

In the next 4 hours, the GCS suddenly dropped and patient showed signs of herniation. A repeat CT of the brain (Figure 3) showed a massive increase in the left extradural bleed with significant midline shift. The patient was immediately taken up for a left parieto-occipital decompressive craniectomy with the evacuation of the EDH under FFP (fresh frozen plasma) cover. After decompression, the brain seemed to be non-pulsatile and oedematous. The GCS did not improve after surgery and the patient was hyperventilated postoperatively. He was given full dose decongestants with pulses of Methyl Prednisolone in lieu of a positive APLA test.

An hour later, pupillary asymmetry re-manifested and a repeat CT scan showed profound diffuse cerebral oedema. (Figure 4) As a last rescue effort, a bilateral large fronto-temporo-parietal decompressive craniectomy was performed, under cover of plasma products and steroids, but to no avail. Post procedure there was no spontaneous respiration and pupils were bilaterally dilated and fixed. The scalp flaps were tense and there was a bloody ooze emanating from the suture line. The patient passed away, later in the night, despite all efforts to resuscitate him.

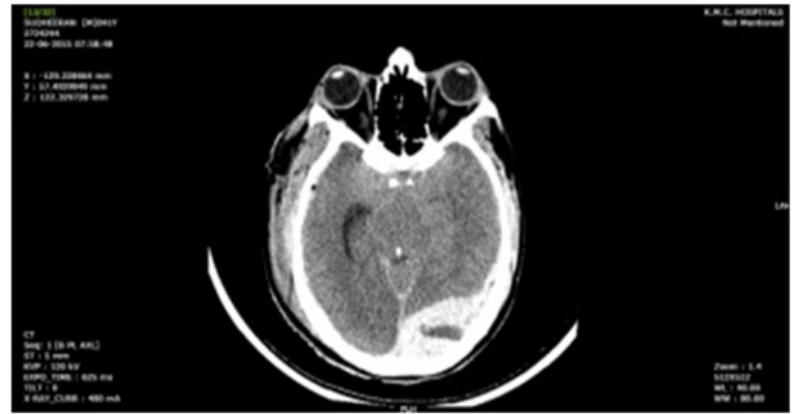

Figure 3 Postoperative Plain, CT Brain showing a significant increase in the left parieto-occipital extradural bleed with significant mass effect and oedema.

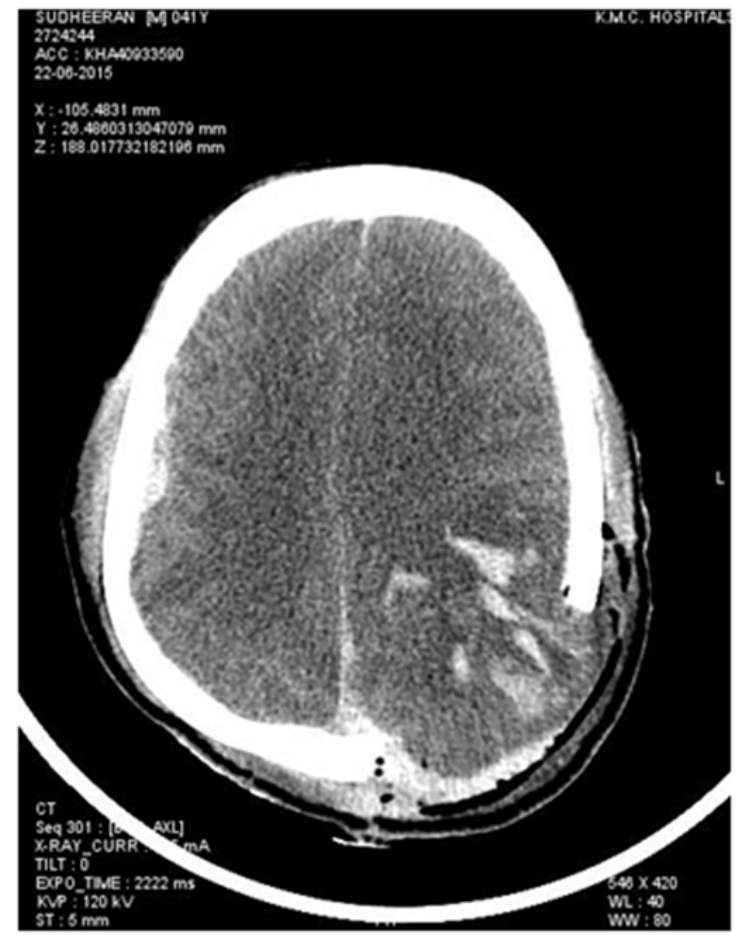

Figure 4 Repeat postoperative scan (post left PO decompressive craniectomy) with significant oedema and mass effect.

\section{Discussion}

Antiphospholipid syndrome (APS) is a multisystem autoimmune disease characterized by a pro-thrombotic state. The Catastrophic Antiphospholipid syndrome is an extreme variant of APS, which is characterized by a cytokine storm, that rapidly moves from a prothrombotic state to consumption coagulopathy leading to severe morbidity and mortality. ${ }^{1}$ CAPS by itself, is not a homogenous monobloc but a spectrum of conditions. ${ }^{2}$ Described by Asherson and classified by Miyasakis et al., ${ }^{2}$ a clinic-immuno-pathological criterion has been postulated for an arriving at a diagnosis (Table $1 \& 2$ ). As per the criteria, our patient had "probable CAPS" as histo-pathological samples could not be taken. ${ }^{3}$

A significant difficulty in the diagnosis of this condition is the time taken for processing investigations and interpreting the data. A similar situation was encountered in our patient and compounded with the rapid neurological deterioration posed by the intracranial bleeds, the eventual diagnosis was futile and the outcome was bleak. 
Table I APLS classification criteria²

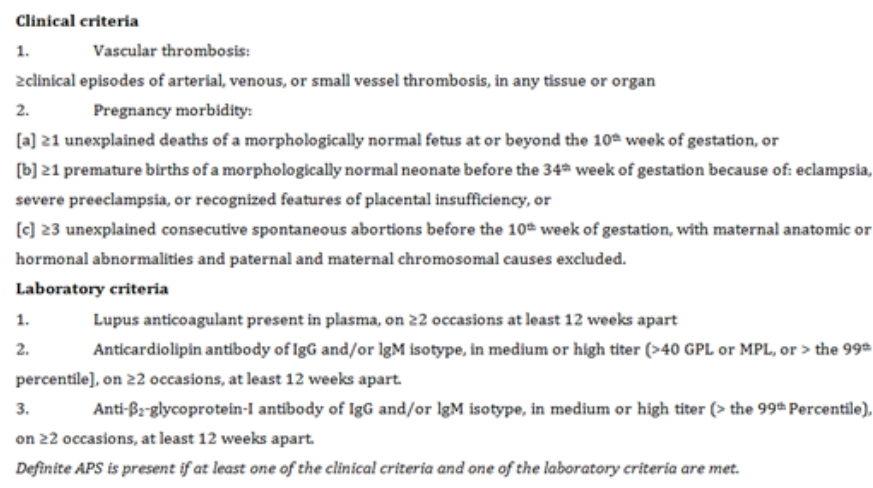

Table 2 Preliminary classification criteria for CAPS ${ }^{*}$ Vasculitis may coexist, but significant thrombosis must be present as well

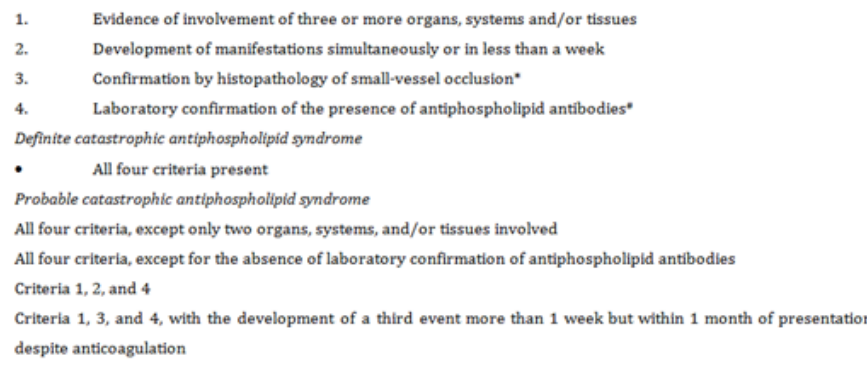

\#"Positive aPL" twice 12 weeks apart (of note, the original Sapporo APS classification criteria required two positive aPL tests 6 weeks apart (Wilson et al. 1999). which has been changed to 12 weeks as part of the updated Sapporo APS classification criteria (Miyakis et al. 2006).

Another dilemma was the interpretation of the APLA titres in this patient. ${ }^{4}$ Transient elevation is often seen in patients with chronic inflammation and sepsis. Various reports in literature have documented cases were APLA was negative, making the interpretation more intriguing. Recommendations for starting steroids, before having a positive or significant APLA titre, have been suggested in view of sudden arrest in the progression of the disease. ${ }^{5}$ The dilemma encountered with this approach is that sepsis, which has a similar presentation to CAPS (transient elevation in APLA titres), is known to flare up with the use of steroids, a potent immuno-modulator.

CAPS and CAPS-like conditions have overlapping features with other connective tissue disorders like TTP, HIT, SLE (Thrombotic thrombocytopenic purpura/ Moschcowitz syndrome, Heparin induced thrombocytopenia, Systemic lupus erythematosis). The presence of Lupus Anticoagulant greatly aids in the diagnosis and treatment of these conditions. ${ }^{6-8}$ This was negative in our patient.

The thrombotic storm, that often manifests in patients with CAPS (Table 3), and associated micro-angiopathic APS carry a very poor prognosis, despite significant advances in therapy and diagnostics. The overall mortality exceeds $50 \%$ despite treatment. ${ }^{1,6-10}$

Much highlight has been given to the presence of triggering stimuli that incite the cascade of thrombosis which eventually resulting in consumption coagulopathy. These triggers can range from fever, stress, dys-electrolytemia, surgery and trauma. ${ }^{1,6-8}$ All these must be avoided to arrest rapid progression, however, in some cases, such as in the present case, we were unable to refrain from surgery to reduce the rapidly progressing ICP. Surgery, though aimed at saving his life, might have been the trigger that caused the rapid decline and caused the eventual fatality in this patient.
Table 3 Clinical characteristics of Thrombotic Storm ${ }^{10}$

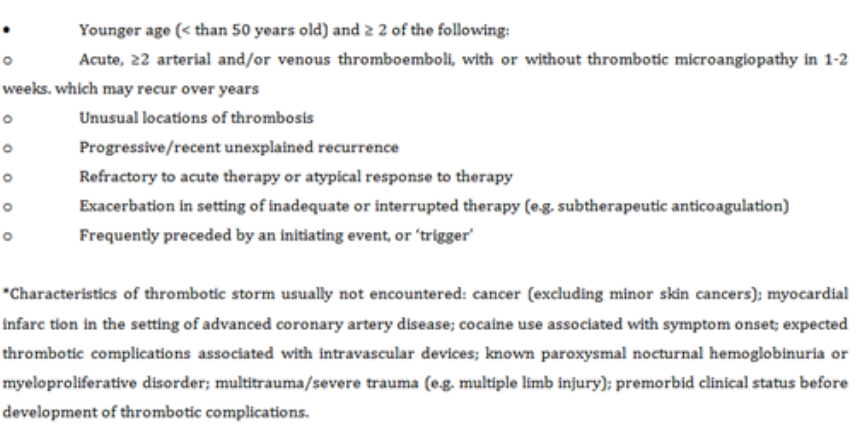

\section{Conclusion}

CAPS is an uncommon yet a devastating event which is associated with a high mortality. Early diagnosis and prompt treatment can halt the progression of this untoward complication and mellow the course of the disease. One should have a high index of suspicion when presented with a patient with multisystem involvement in the background of spontaneous thrombotic episodes. Once an APLA titer is positive, prompt usage of steroids with avoidance of triggers must be given a high priority to prevent the thrombotic storm that ensues. Nonadherence to these recommendations leads to rapid deterioration and inevitable death of the patient.

\section{Acknowledgments}

None.

\section{Conflicts of interest}

None.

\section{References}

1. Aguiar CL, Erkan D. Catastrophic antiphospholipid syndrome: how to diagnose a rare but highly fatal disease. Ther Adv Musculoskelet Dis. 2013;5(6):305-314.

2. Miyakis S, Lockshin MD, Atsumi T, et al. International consensus statement on an update of the classification criteria for definite antiphospholipid syndrome (APS). J Thromb Haemost. 2006;4(2):295306

3. Asherson RA, Cervera R, de Groot PG, et al. Catastrophic antiphospholipid syndrome: international consensus statement on classification criteria and treatment guidelines. Lupus. 2003;12(7):530534.

4. Asherson R., Pierangeli S., Cervera R. Microangiopathicantiphospholipid associated syndromes revisited new concepts relating to antiphospholipid antibodies and syndromes. J Rheumatol. 2007;34(9):1793-1795.

5. Vora SK, Asherson RA, Erkan D. Catastrophic antiphospholipid syndrome. J Intensive Care Med. 2006;21(3):144-159.

6. Erkan D, Yazici Y, Lockshin M. Catastrophic antiphospholipid syndrome (CAPS) or antiphospholipid syndrome (APS) with a catastrophic event. Lupus. 2002;11:617.

7. Erkan D, Yazici Y, Peterson MG, et al. A cross sectional study of clinical thrombotic risk factors and preventive treatments in antiphospholipid syndrome. Rheumatology (Oxford). 2002;41(8):924-929.

8. Girón-González JA, García del Río E, Antiphospholipid syndrome and asymptomatic carriers of antiphospholipid antibody: prospective analysis of 404 individuals. J Rheumatol. 2004;31(8):1560-1567. 
9. Cervera R, Font J, Gómez-Puerta JA, et al. Validation of the preliminary criteria for the classification of catastrophic antiphospholipid syndrome. Ann Rheum Dis. 2005;64(8):1205-1209.
10. Kitchens CS, Erkan D, Brandão LR, et al. Thrombotic storm revisited: preliminary diagnostic criteria suggested by the Thrombotic Storm Study Group. Am J Med. 2011;124(4):290-296. 